\title{
Endocrine function and gland volume after endoscopic transsphenoidal surgery for nonfunctional pituitary macroadenomas
}

\author{
Maya Harary, BA, ${ }^{1}$ Aislyn C. DiRisio, BS, ${ }^{1,2}$ Hassan Y. Dawood, BS, ${ }^{1}$ John Kim, MD, ${ }^{3}$ \\ Nayan Lamba, BA, ${ }^{1}$ Charles H. Cho, MD, MBA, ${ }^{4}$ Timothy R. Smith, MD, ${ }^{1,5}$ Hasan A. Zaidi, MD,,${ }^{1,5}$ and \\ Edward R. Laws Jr., MD 5 \\ ${ }^{1}$ Computational Neuroscience Outcomes Center (CNOC), Department of Neurosurgery, ${ }^{4}$ Department of Radiology, and ${ }^{5}$ Pituitary \\ and Neuroendocrine Center, Department of Neurosurgery, Brigham and Women's Hospital, Harvard Medical School, Boston, \\ Massachusetts; ${ }^{2}$ cahn School of Medicine at Mount Sinai, New York City, New York; and ${ }^{3}$ Division of Neuroradiology, University \\ of Michigan, Ann Arbor, Michigan
}

OBJECTIVE Loss of pituitary function due to nonfunctional pituitary adenoma (NFPA) may be due to compression of the pituitary gland. It has been proposed that the size of the gland and relative perioperative gland expansion may relate to recovery of pituitary function, but the extent of this is unclear. This study aims to assess temporal changes in hormonal function after transsphenoidal resection of NFPA and the relationship between gland reexpansion and endocrine recovery.

METHODS Patients who underwent endoscopic transsphenoidal surgery by a single surgeon for resection of a nonfunctional macroadenoma were selected for inclusion. Patients with prior pituitary surgery or radiosurgery were excluded. Patient characteristics and endocrine function were extracted by chart review. Volumetric segmentation of the pre- and postoperative ( $\geq 6$ months) pituitary gland was performed using preoperative and long-term postoperative MR images. The relationship between endocrine function over time and clinical attributes, including gland volume, were examined.

RESULTS One hundred sixty eligible patients were identified, of whom $47.5 \%$ were female; $56.9 \%$ of patients had anterior pituitary hormone deficits preoperatively. The median tumor diameter and gland volume preoperatively were 22.5 $\mathrm{mm}$ (interquartile range [IQR] $18.0-28.8 \mathrm{~mm}$ ) and $0.18 \mathrm{~cm}^{3}$ (IQR $\left.0.13-0.28 \mathrm{~cm}^{3}\right)$, respectively. In $55 \%$ of patients, endocrine function normalized or improved in their affected axes by median last clinical follow-up of 24.4 months (IQR 3.251.2 months). Older age, male sex, and larger tumor size were associated with likelihood of endocrine recovery. Median time to recovery of any axis was 12.2 months (IQR 2.5-23.9 months); hypothyroidism was the slowest axis to recover. Although the gland significantly reexpanded from preoperatively $\left(0.18 \mathrm{~cm}^{3}, I Q R 0.13-0.28 \mathrm{~cm}^{3}\right)$ to postoperatively $(0.33$ $\mathrm{cm}^{3}$, IQR $0.23-0.48 \mathrm{~cm}^{3} ; \mathrm{p}<0.001$ ), there was no consistent association with improved endocrine function.

CONCLUSIONS Recovery of endocrine function can occur several months and even years after surgery, with more than $50 \%$ of patients showing improved or normalized function. Tumor size, and not gland volume, was associated with preserved or recovered endocrine function.

https://thejns.org/doi/abs/10.3171/2018.5.JNS181054

KEYWORDS nonfunctional pituitary adenoma; transsphenoidal surgery; endoscopic surgery; endocrine status; volumetric segmentation; pituitary surgery

$\mathrm{N}$ ONFUNCTIONAL pituitary adenomas (NFPAs) represent about one-third of pituitary adenomas..$^{16,17}$ NFPAs are histologically divided into null cell adenomas and silent hormone-staining adenomas. ${ }^{25}$ Given the absence of hormone secretion, NFPAs typically can be incidentally discovered or present with symptoms re- lated to mass effect on adjacent neurovascular structures. ${ }^{23}$ Namely, they may present with headache due to irritation of the dura, vision changes due to compression of overlying visual apparatus, and hypopituitarism from compression of the pituitary gland and/or stalk.

Dysfunction in one or more anterior pituitary axes has

ABBREVIATIONS BWH = Brigham and Women's Hospital; GH = growth hormone; HRT = hormone replacement therapy; IQR = interquartile range; NFPA = nonfunctional pituitary adenoma.

SUBMITTED April 19, 2018. ACCEPTED May 29, 2018.

INCLUDE WHEN CITING Published online November 9, 2018; DOI: 10.3171/2018.5.JNS181054. 
been reported in up to $85 \%$ of NFPAs,${ }^{19}$ and the presence of hypopituitarism can be an indication for surgical intervention, even in the absence of visual deficits or obstructive hydrocephalus. ${ }^{21}$ Although several studies have reported on the rates of preoperative endocrine dysfunction and the likelihood of recovery, $4,7,10,19$ few have investigated the predictive value of the size of the gland and stalk, or how the extent of gland reexpansion relates to functional recovery.

Two previously published studies have reported the relationship between gland volume and endocrine recovery up to 1 year following resection of NFPAs, showing variable results. ${ }^{6}, 13$ Both studies included a heterogeneous cohort of patients treated by either a microscopic or endoscopic transsphenoidal approach, and included patients treated for a recurrence following surgery or radiosurgery. Cho et al. ${ }^{6}$ specifically included both NFPAs and secretory adenomas, and defined endocrine dysfunction only by the requirement of hormone replacement therapy (HRT), regardless of serum hormone levels.

In this study we aimed to characterize the relationship between gland volume and endocrine status pre- and postoperatively at long-term follow-up among a more homogeneous cohort of patients. To minimize the impact of confounding factors, we chose to include only patients with macroadenomas $(\geq 10 \mathrm{~mm})$ treated with an endoscopic transsphenoidal approach, and excluded patients with prior pituitary intervention, including surgery and radiosurgery. In addition to volumetric analysis of the gland, we performed a time-to-event analysis to delineate the time to recovery of the individual anterior pituitary axes. These data may provide prognostic information regarding the likelihood and timing of postoperative endocrine recovery.

\section{Methods \\ Patient Selection}

Departmental records for the Department of Neurosurgery at Brigham and Women's Hospital (BWH) were screened for patients who underwent an endoscopic transsphenoidal surgery between 2008 and 2016 for an NFPA by a single surgeon (E.R.L.). NFPA was defined based on the absence of anterior pituitary hormone hypersecretion, based on both serum hormone tests (see reference values below) and the absence of clinical signs and symptoms associated with hypersecretion. Diagnosis of NFPA was confirmed on surgical pathology. Both null cell adenomas and clinically silent hormone-staining adenomas were included. Only macroadenomas were included, as defined by a widest tumor diameter $\geq 10 \mathrm{~mm}$ on any plane in preoperative imaging. Exclusion criteria were 1) prior pituitary surgery or radiation, 2) the use of a combined endoscopicmicroscopic approach, and 3) postoperative radiosurgery. This study was IRB-approved.

\section{Preoperative Endocrine Evaluation}

Endocrine status information was extracted from patient charts to assess anterior and posterior pituitary function. Anterior pituitary dysfunction was defined as the presence of hypothyroidism, adrenal insufficiency, hypo- gonadism, and/or growth hormone (GH) deficiency. For each anterior pituitary axis, dysfunction was defined as low serum hormone levels according to the reference levels described below, or the need for HRT. Three patients had preexisting primary hypothyroidism and were not considered as deficient for that axis. Patients who received perioperative steroids explicitly for the relief of edema and protection of the visual apparatus were not considered as having adrenal insufficiency. The majority of endocrine laboratory tests were conducted at the BWH laboratories with the reference ranges listed below. For patients who had laboratory tests performed at outside laboratories, the reference ranges used by those facilities were used to interpret the laboratory result. Hypothyroidism was defined as a low free thyroxine level (T4, reference range $0.8-1.8 \mathrm{mIU} / \mathrm{ml}$ ) with a normal or low thyroid-stimulating hormone level (reference range 0.5-5.0 $\mathrm{mIU} / \mathrm{ml}$ ). Hypoadrenalism, or adrenal insufficiency, was defined as low cortisol (reference range at 8 AM: $6.2-19.4 \mathrm{mg} / \mathrm{dl}$; at 4 PM: $2.3-11.9 \mathrm{mg} / \mathrm{dl}$ ) with or without low corticotropin (reference range $10-60 \mathrm{pg} / \mathrm{dl}$ ). A cosyntropin stimulation test was performed at the clinician's discretion. GH deficiency was defined as low insulin-like growth factor-1 or below normal limit GH; both these tests were performed at outside laboratories and were interpreted according to the performing laboratory's reference ranges at the time of testing. Male hypogonadism was defined as low serum testosterone (reference range 193-740 ng/dl).

Women $\leq 45$ years old were assumed to be premenopausal, and women $\geq 55$ years old were assumed to be postmenopausal. For women between the ages of 46 and 54 , the clinic notes were assessed for a diagnosis of menopause. In premenopausal women, female hypogonadism was defined as low estradiol levels (reference range: follicular $14-188 \mathrm{pg} / \mathrm{ml}$, ovulatory $75-364 \mathrm{pg} / \mathrm{ml}$, luteal 41-177 pg/ml), in the presence of low or normal follicular stimulating hormone (reference range: follicular phase 3.9-13.2 mIU/L, midcycle 4.2-18.4 mIU/L, luteal phase $1.7-10.4 \mathrm{mIU} / \mathrm{L}$ ) or luteal hormone (reference range: follicular phase 2.4-12.6 mIU/L, midcycle 14.0-95.6 mIU/L, luteal phase 1.0-11.4 mIU/L). Postmenopausal women were excluded from all analyses pertaining to female gonadal function. Prior to August 2011, elevated prolactin level was defined as $>26.7 \mathrm{ng} / \mathrm{ml}$ (women $<50$ years old), $>19.6 \mathrm{ng} / \mathrm{ml}$ (women $>50$ years old), and > $15.2 \mathrm{ng} / \mathrm{ml}$ (men). Thereafter, hyperprolactinemia was defined as $>$ $23.3 \mathrm{ng} / \mathrm{ml}$ (nonpregnant women) and $>13.1 \mathrm{ng} / \mathrm{ml}$ (men). Diabetes insipidus was defined clinically and/or by the need for desmopressin therapy.

\section{Postoperative Endocrine Evaluation}

Recovery of anterior pituitary hormone axes was defined as 1) the return to reference range serum levels in a patient not receiving HRT, or 2) cessation of HRT. In the latter group, if the patient tolerated the cessation of HRT, laboratory confirmation of the normalization of serum levels was not required for the axis to be considered recovered. Time of recovery was defined as either the date of the normal serum laboratory study or the date referred to in the neurosurgery or endocrinology note as date of recovery. Normalization of anterior pituitary function was 
defined as resolution of all preoperative deficits, while improvement in function was defined as resolution of some but not all preoperative deficits. Local patients followed at our institution received neuroendocrine follow-up at 6 weeks, 3 months, and 1 year after surgery, with more frequent or further visits as needed. In cases in which travel to our institution was a significant barrier, patients were followed by their home endocrinologist according to their practice, with laboratory results and clinical status typically communicated back to us.

New postoperative deficits were defined in the same manner as described for preoperative deficits. Length of clinical follow-up was defined as time between surgery and the last visit at BWH neurosurgery or endocrinology.

\section{MRI Acquisition and Analysis}

Preoperative widest tumor diameter in any plane, as recorded in the radiology report, was collected for all patients. If a report was not available, the diameter was measured by a single investigator. Giant adenomas were defined as those measuring $\geq 4 \mathrm{~cm} \cdot{ }^{18,21,24}$

Inclusion criteria for volumetric analysis were the availability of contrast-enhanced T1-weighted coronal or sagittal MRI sequences both preoperatively and at longterm follow up at $\geq 6$ months postoperatively. Patients with appropriate imaging were excluded from volumetric analysis if the gland was not discernable on preoperative scans $(\mathrm{n}=5)$, or if the long-term follow-up MRI showed obvious recurrent or residual tumor $(n=11)$. These exclusions were made based on the neuroradiology imaging report and/or with secondary review by a neurosurgeon (H.A.Z.). Radiological follow-up time was defined as the time between surgery and the postoperative imaging study used for segmentation purposes.

Volumetric segmentation of the pituitary gland on both preoperative and postoperative MR images was performed using the open-source software 3D Slicer (version 4.8; https://www.slicer.org/) by two investigators (M.H. and J.K.). The pituitary gland was identified by following the stalk of the pituitary anteroventrally. Segmentation was primarily performed on the coronal sequence. If the stalk was not visible on the coronal sequence, the sagittal sequence was used instead.

In order to define deviation of the pituitary stalk, a vertical line was drawn on the coronal plane originating from the ventricular septum pellucidum, crossing through the center of the optic chiasm and terminating at the bottom of the sella. If at the point of insertion into the gland, the stalk transected that line, the stalk position was defined as midline, otherwise it was defined as deviated. Stalk diameter was defined at its widest point, typically close to its origin.

\section{Statistical Analysis}

Statistical analysis was conducted using $\mathrm{R}$ software (version 3.4.0; https://www.r-project.org). A Student t-test and a Wilcoxon-Mann-Whitney U-test were used to compare continuous parametric and nonparametric variables, respectively. A paired Wilcoxon-Mann-Whitney U-test was used to compare pre- and postoperative gland vol-
TABLE 1. Patient characteristics $(n=160)$

\begin{tabular}{lc}
\hline \multicolumn{1}{c}{ Variable } & Value \\
\hline Mean age, yrs (SD) & $57.1(13.5)$ \\
\hline No. of females (\%) & $76(47.5)$ \\
\hline Premenopausal females (\%) & $28(36.8)$ \\
\hline Incidental diagnosis (\%) & $54(33.8)$ \\
\hline Median tumor diameter, mm (IQR) & $22.50(18.00-28.77)$ \\
\hline Giant adenoma, $\geq 4 \mathrm{~cm}(\%)$ & $10(6.2)$ \\
\hline Hyperprolactinemia (\%) & $77(48.1)$ \\
\hline Any symptoms at time of diagnosis, $\mathrm{n}(\%)$ & $123(76.9)$ \\
\hline Headache & $55(34.4)$ \\
\hline Endocrine-related & $48(30.0)$ \\
\hline Vision & $67(41.9)$ \\
\hline Any anterior pituitary endocrine deficit & \\
\hline preop, $n$ (\%) & $69(43.1)$ \\
\hline No deficiencies & $91(56.9)$ \\
\hline 1 or more deficiencies & \\
\hline Endocrinopathies, $n$ (\%) & $52(32.5)$ \\
\hline Hypothyroidism & $35(21.9)$ \\
\hline Adrenal insufficiency & $40(47.6)$ \\
\hline Male hypogonadism & $6(21.4)$ \\
\hline Female hypogonadism in premenopausal \\
females
\end{tabular}

$\mathrm{Pt}=$ patient.

ume. A chi-square test was used to compare categorical variables. Bivariate and multivariable logistic regression was performed using generalized linear models. Linear regression was used to assess relationships between gland volume, age, and tumor size. Kaplan-Meier time-to-event analysis was conducted using the survival package with a log-rank statistic. To identify the threshold values of age and tumor size that best discriminated the likelihood of postoperative endocrine recovery, we maximized the Youden Index $(\mathrm{J})$, which equally weighs both sensitivity and specificity. A p value $<0.05$ was considered statistically significant.

\section{Results}

\section{Baseline Patient Characteristics}

A total of 160 patients were identified (Table 1). Of the included patients, $47.5 \%$ were women and the mean age at the time of surgery was $57.1 \pm 13.5$ years. Although in $40.6 \%$ of cases the NFPA was discovered incidentally, $76.9 \%$ of patients reported symptoms attributable to the 


\section{Pre-op endocrine deficits}
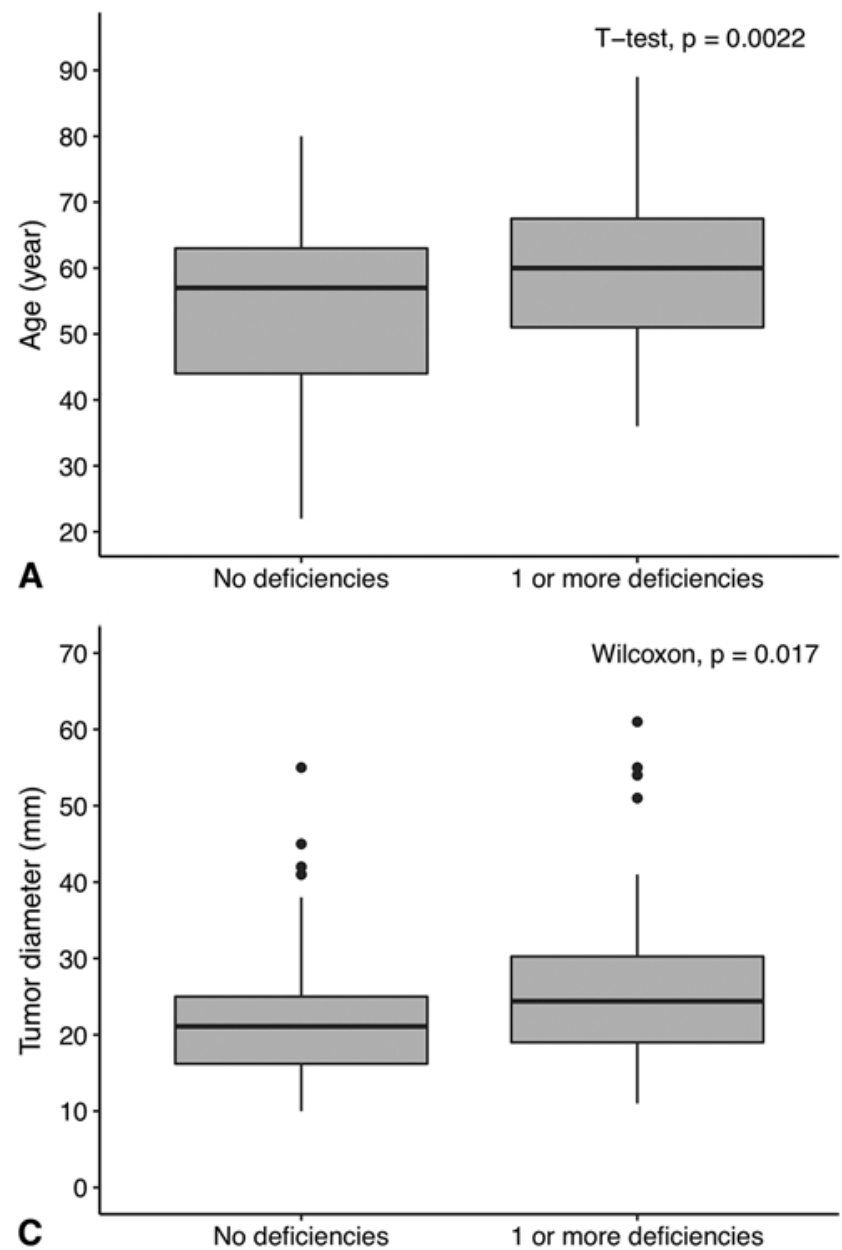

Post-op endocrine recovery
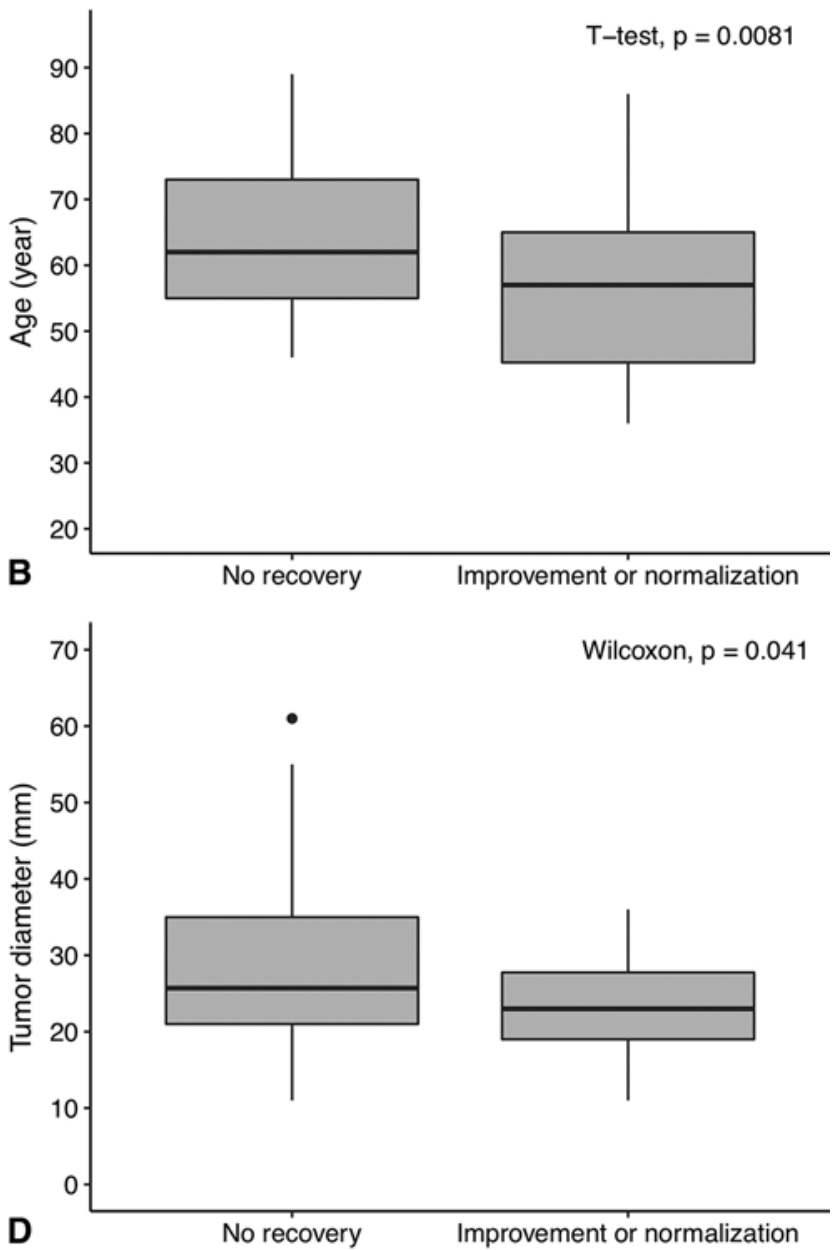

FIG. 1. Box-and-whisker plots. A and C: Association between the presence of any preoperative anterior pituitary endocrine deficiency and age $(A)$ and tumor diameter $(C)$. B and $D$ : Association between the recovery of function in any anterior pituitary endocrine axis and age (B) and tumor diameter (D). No recovery = none of the preoperative deficits resolved by last follow-up; improvement or normalization $=$ resolution of at least 1 preoperative deficit.

NFPA once they came to neurosurgical attention. These symptoms included visual disturbance $(41.9 \%)$, headache (34.4\%), and endocrine-related symptoms (30.0\%). The median NFPA diameter was $22.5 \mathrm{~mm}$ (interquartile range [IQR] 18.0-28.8 mm, range $10.0-61.0 \mathrm{~mm}$ ) with $6.2 \%$ of patients having a giant NFPA $(\geq 4 \mathrm{~cm})$.

\section{Preoperative Endocrine Status}

Preoperatively, $56.9 \%$ of patients had at least one anterior pituitary endocrine deficit, and $26 \%$ had 2 or more deficits. The most common endocrinopathy was hypothyroidism $(32.5 \%)$, followed by male hypogonadism $(47.6 \%$ of men), and adrenal insufficiency $(21.9 \%$, Table 1$)$. At the time of presentation, $36.8 \%$ of the women were premenopausal; of these, $21.4 \%$ had female hypogonadism. Of the 40 men and 6 women with hypogonadism, $52.5 \%$ and $66.7 \%$ were hyperprolactinemic, respectively.

Both older age $(\mathrm{p}=0.002)$ and larger tumor diameter $(\mathrm{p}$ $=0.02$ ) were significantly associated with the presence of preoperative anterior pituitary dysfunction (Fig. 1). When an axis-by-axis evaluation was performed, larger tumor size was only associated with the presence of hypothyroidism ( $p=0.001)$ and not with deficiency in any of the other anterior pituitary axes. Similarly, older age was not strongly associated with deficiency in any one axis in particular. Men were more likely to have preoperative deficiency ( $p$ $=0.005$ ). This is likely due to the contribution of male hypogonadism, as there was no significant difference in the incidence of deficits in other axes between the sexes. Gland volume was not significantly associated with the presence of preoperative endocrine deficits (Table 2). On multivariable analysis, incorporating age, sex, and tumor diameter, both older (OR 1.04, 95\% CI 1.02-1.05, $\mathrm{p}=0.01)$ and male patients (OR 2.49, 95\% CI 1.77-3.50, $\mathrm{p}=0.01$ ) were more likely to have a preoperative deficit, controlling for the other included variables (Table 2). After controlling for age and sex, tumor diameter was no longer significantly associated with the presence of preoperative deficits. 


\section{Postoperative Hormonal Axis Recovery}

The median postoperative clinical follow-up was 24.4 months (IQR 3.2-51.2 months, range < 1-267 months). Of the 91 patients with preoperative endocrine deficits, postoperative endocrine function was either completely normalized (37.4\%), improved (17.6\%), or not improved (45.1\%, Table 3). The likelihood of recovery varied by axis. The preoperative deficit most likely to recover after surgery was hypoadrenalism (62.9\% recovery). Preoperative hypothyroidism was persistent in $73.1 \%$ of cases. Of the 6 women with preoperative hypogonadism, all those with hyperprolactinemia $(n=4)$ showed resolution of hypogonadism, while those with normal preoperative prolactin levels $(n=2)$ had persistence of hypogonadism at last follow-up. This trend was not observed in the male patients, in whom the presence of preoperative hyperprolactinemia was not significantly associated with resolution of hypogonadism. Hypothyroidism was more likely to recover in hyperprolactinemic patients $(\mathrm{p}=0.04)$.

Both younger age $(\mathrm{p}=0.008)$ and smaller tumor diameter $(p=0.04)$ were associated with improvement in anterior pituitary function postoperatively (Fig. 1). Males were significantly more likely to have improvement in pituitary function $(\mathrm{p}=0.02)$. The pre- and postoperative volume of the gland were not significantly associated with resolution of hypopituitarism, nor was the percentage change in volume (Table 2). On multivariable analysis, incorporating age, sex, and tumor diameter, older age (OR $0.95,95 \%$ CI $0.93-0.97, \mathrm{p}=0.01$ ) and larger tumor diameter (OR 0.94, $95 \%$ CI $0.91-0.97, \mathrm{p}=0.03$ ) were associated with lower likelihood of postoperative endocrine function recovery, controlling for the other included variables (Table 2). A tumor diameter of $28.7 \mathrm{~mm}$ and an age of 47 years were the best threshold values to discriminate patients' likelihood of postoperative anterior pituitary function recovery, albeit with modest area under the curve $(0.625$ and 0.655 , respectively). Men were more likely to experience endocrine recovery relative to women (OR 3.41, 95\% CI 2.06-5.64, p $=0.02$ ), controlling for age and tumor diameter.

The median time to the earliest recovery of any axis was 12.2 months (IQR 2.5-23.9 months, Fig. 2A). On an axis-by-axis analysis, hypothyroidism was significantly slower to recover than both adrenal insufficiency and hypogonadism (Fig. 2B). There was no significant difference in the time to recovery among the remaining endocrine deficits.

\section{New Postoperative Deficits}

Thirty-seven patients (23.1\%) had new postoperative endocrine deficits, including diabetes insipidus (Table 4). Consistent with previous studies, $51.4 \%$ of these 37 patients had preoperative deficits in other axes..$^{15}$ Although $7.5 \%$ of patients $(n=12)$ developed diabetes insipidus postoperatively, this change was typically transient $(\mathrm{n}=$ 8 ) and resolved within 1 month after surgery. Four patients had diabetes insipidus persisting to a median of 26.8 months (IQR 17.9-32.3 months) after surgery. Adrenal insufficiency was the most common new deficit, occurring at a rate of $16.4 \%$, with most cases being transient and resolving at a median of 2.5 months (IQR 0.6-12.2 months) postoperatively. New hypothyroidism occurred at a rate of $9.3 \%$ with all cases persisting to last followup at a median of 38.3 months (IQR 19.9-70.0 months). Similarly, new GH deficiency occurred at a rate of $2.8 \%$ and all cases were persistent (62.3 months, IQR 51.0-74.0 months). There were 3 cases of new male hypogonadism (6.8\%); of those, one recovered at 20.9 months postoperatively, and the remaining 2 cases persisted to last clinical follow-up (54.2 and 77.9 months). There were no cases of new female hypogonadism.

On bivariate analysis, both larger postoperative gland volume (OR $0.01,95 \%$ CI $0.00-0.06, \mathrm{p}=0.008)$ and female sex (OR $0.37,95 \%$ CI $0.24-0.57, \mathrm{p}=0.02$ ) were associated with lower odds of having a new postoperative anterior pituitary endocrine deficit. Age, tumor size, presence of preoperative deficits, and relative expansion of the gland were not associated with the incidence of new anterior pituitary deficits. On multivariable analysis, only larger postoperative gland volume remained significantly associated with the lower odds of having a new anterior pituitary deficit (OR $0.01,95 \%$ CI $0.00-0.06, \mathrm{p}=0.009$ ), controlling for sex. Neither patient demographics, nor tumor size, nor gland size were predictive of postoperative diabetes insipidus.

\section{Volumetric Analysis}

Of 160 patients, $72(45 \%)$ met criteria for inclusion in volumetric analysis with median radiographic follow-up of 30.7 months (IQR 18.6-44.1 months, range 8.7-93.4 months). Reasons for exclusion were the absence of longterm ( $\geq 6$ months postoperative) imaging (45\%), the presence of obvious recurrent or residual tumor on long-term follow-up imaging (7\%), and the inability to reliably discern the normal gland preoperatively (3\%). In this group, median preoperative gland volume was $0.18 \mathrm{~cm}^{3}$ (IQR $0.13-0.28 \mathrm{~cm}^{3}$, Table 5). The gland significantly expanded postoperatively, reaching a median volume of $0.33 \mathrm{~cm}^{3}$ (IQR $0.23-0.48 \mathrm{~cm}^{3}, \mathrm{p}<0.001$ ). There was significant interpatient variability in gland volume both pre- and postoperatively $(\mathrm{p}<0.001)$. Bivariate analysis showed that neither age nor tumor size were associated with gland volume pre- or postoperatively, nor with gland expansion (all $\mathrm{p}>$ $0.05)$. Prior to surgery, the stalk was deviated in $66.2 \%$ of the patients. In $59.6 \%$ of these patients, the stalk returned to midline position postoperatively. None of the patients had new postoperative deviation of the stalk. Neither preoperative stalk deviation nor its correction was significantly associated with endocrine status (all $\mathrm{p}>0.05$ ). Sample cases are shown in Fig. 3.

\section{Discussion}

Sellar pathology can affect endocrine function either by hypersecretion of hormones by functional tumors or by mechanical compression of the pituitary gland and stalk, leading to decreased production and secretion of pituitary hormones. NFPAs cause deficits via the latter mechanism, with mass effect leading to a deficit either in a single hormonal axis or, more rarely, panhypopituitarism. ${ }^{14}$ This is attributed to disruption of delicate hypophyseal vasculature that can disrupt both hypothalamic stimulating and inhibiting factor delivery and can also cause gland ische- 
TABLE 2. Predictors of anterior pituitary endocrine function

\begin{tabular}{|c|c|c|c|c|}
\hline \multirow{2}{*}{$\begin{array}{c}\text { Predictors of Anterior Pituitary } \\
\text { Endocrine Deficiency }\end{array}$} & \multicolumn{2}{|l|}{ Bivariate } & \multicolumn{2}{|c|}{ Multivariable } \\
\hline & OR $(95 \% \mathrm{Cl})$ & $p$ Value & OR $(95 \% \mathrm{Cl})$ & $\mathrm{p}$ Value \\
\hline \multicolumn{5}{|l|}{ Preop } \\
\hline Age, yr & $1.04(1.03-1.05)$ & 0.003 & $1.04(1.02-1.05)$ & 0.01 \\
\hline Sex (ref: female) & $2.61(1.88-3.62)$ & 0.004 & $2.49(1.77-3.50)$ & 0.01 \\
\hline Tumor diameter, mm & $1.04(1.02-1.06)$ & 0.04 & $1.03(1.01-1.05)$ & 0.11 \\
\hline Hyperprolactinemia (ref: no) & $1.22(0.88-1.68)$ & 0.54 & & \\
\hline Preop gland vol, $\mathrm{cm}^{3}$ & $0.07(0.01-0.4)$ & 0.13 & & \\
\hline \multicolumn{5}{|l|}{ Postop } \\
\hline Age, yr & $0.96(0.94-0.97)$ & 0.01 & $0.95(0.93-0.97)$ & 0.01 \\
\hline Sex (ref: female) & $2.99(1.91-4.68)$ & 0.02 & $3.41(2.06-5.64)$ & 0.02 \\
\hline Tumor diameter, $\mathrm{mm}$ & $0.94(0.91-0.96)$ & 0.01 & $0.94(0.91-0.97)$ & 0.03 \\
\hline Hyperprolactinemia (ref: no) & $0.94(0.91-0.96)$ & 0.47 & & \\
\hline Preop gland vol, $\mathrm{cm}^{3}$ & $200.73(3.15-12808.86)$ & 0.20 & & \\
\hline Postop gland vol, $\mathrm{cm}^{3}$ & $0.66(0.13-3.31)$ & 0.79 & & \\
\hline$\%$ change in gland vol & $1(1-1)$ & 0.22 & & \\
\hline
\end{tabular}

Ref $=$ reference.

Boldface type indicates statistical significance.

mia., ${ }^{3,15,27}$ Tumor resection can subsequently permit restoration of circulation, and give the gland the opportunity to reexpand. It remains unclear, however, how the extent of postoperative gland expansion affects endocrine recovery. In this study we evaluated the clinical and volumetric factors that contribute to preoperative endocrine status and postoperative recovery at long-term follow up.

\section{Endocrine Function}

There is a differential susceptibility to compression among the cell populations of the adenohypophysis, with gonadotropes and thyrotropes being the most susceptible..$^{12,14,27}$ In this study, the most common deficits were indeed hypothyroidism and male hypogonadism, affecting almost $50 \%$ of included males, similar to prior studies., ${ }^{4,7}$ 10,13,19,27 The rates of female hypogonadism were consider-

TABLE 3. Resolution of preoperative endocrine deficits

\begin{tabular}{|c|c|}
\hline Endocrine Deficits & No. of Pts $(\%)$ \\
\hline \multicolumn{2}{|c|}{$\begin{array}{l}\text { Overall anterior pituitary function* (\% of pts w/ any } \\
\text { preop deficiency) }\end{array}$} \\
\hline Normalized & $34(37.4)$ \\
\hline Improved & $16(17.6)$ \\
\hline Not improved & $41(45.1)$ \\
\hline Resolution of hypothyroidism & $14 / 52(26.9)$ \\
\hline Resolution of adrenal insufficiency & $22 / 35(62.9)$ \\
\hline Resolution of male hypogonadism & $19 / 40(47.5)$ \\
\hline Resolution of female hypogonadism & $4 / 6(66.7)$ \\
\hline Resolution of GH deficiency & 9/17 (52.9) \\
\hline Resolution of diabetes insipidus & $1(100.0)$ \\
\hline
\end{tabular}

* Normalization = resolution of all preoperative deficits; improvement = resolution of one, but not all, preoperative deficits; no improvement = none of the preoperative deficits resolved by last follow-up. ably lower.,13 As previously shown, patient demographics were associated with endocrine status, with older, male patients having a higher likelihood of preoperative endocrine deficits. ${ }^{13,19}$

In the postoperative period, the absence of endocrine recovery suggests possible ischemic necrosis to at least some cell populations that may preclude clinically significant recovery of the affected axis. In this study, the deficits most likely to recover were GH deficiency and adrenal insufficiency. Multivariable analysis demonstrated that younger age, smaller tumor size, and male patients were associated with likelihood of postoperative endocrine recovery.

It had been suggested that preoperative hyperprolactinemia is a positive prognostic factor for endocrine recovery, as it may implicate stalk compression and impeded hypothalamic hormone delivery as the major cause of endocrine dysfunction, as opposed to gland ischemia. ${ }^{1,2,19} \mathrm{In}$ this study, this pattern was noted only in some axes. All women in whom hypogonadism resolved were hyperprolactinemic preoperatively. Similarly, resolution of hypothyroidism was associated with preoperative hyperprolactinemia. In hyperprolactinemic patients, hypothyroidism resolved around the reported time of hyperprolactinemia resolution (10.5 vs 12 weeks), while resolution of female hypogonadism lagged 8 weeks behind. ${ }^{28}$ This observed association between hyperprolactinemia and recovery may aid the prognostication of likelihood and time to recovery of hypothyroidism as well as female hypogonadism.

In the time-to-event analysis, we demonstrated that recovery of endocrine function can occur several months and even years after surgery, with more than $50 \%$ of patients showing improved or normalized function. Hypothyroidism was both the slowest to recover and the most persistent deficit. Similar to previous studies, we defined recovery as either normalization of serum hormone levels and/or the cessation of HRT., ${ }^{4,6,7}$ In those patients who required HRT, the decision to attempt a trial off HRT was 

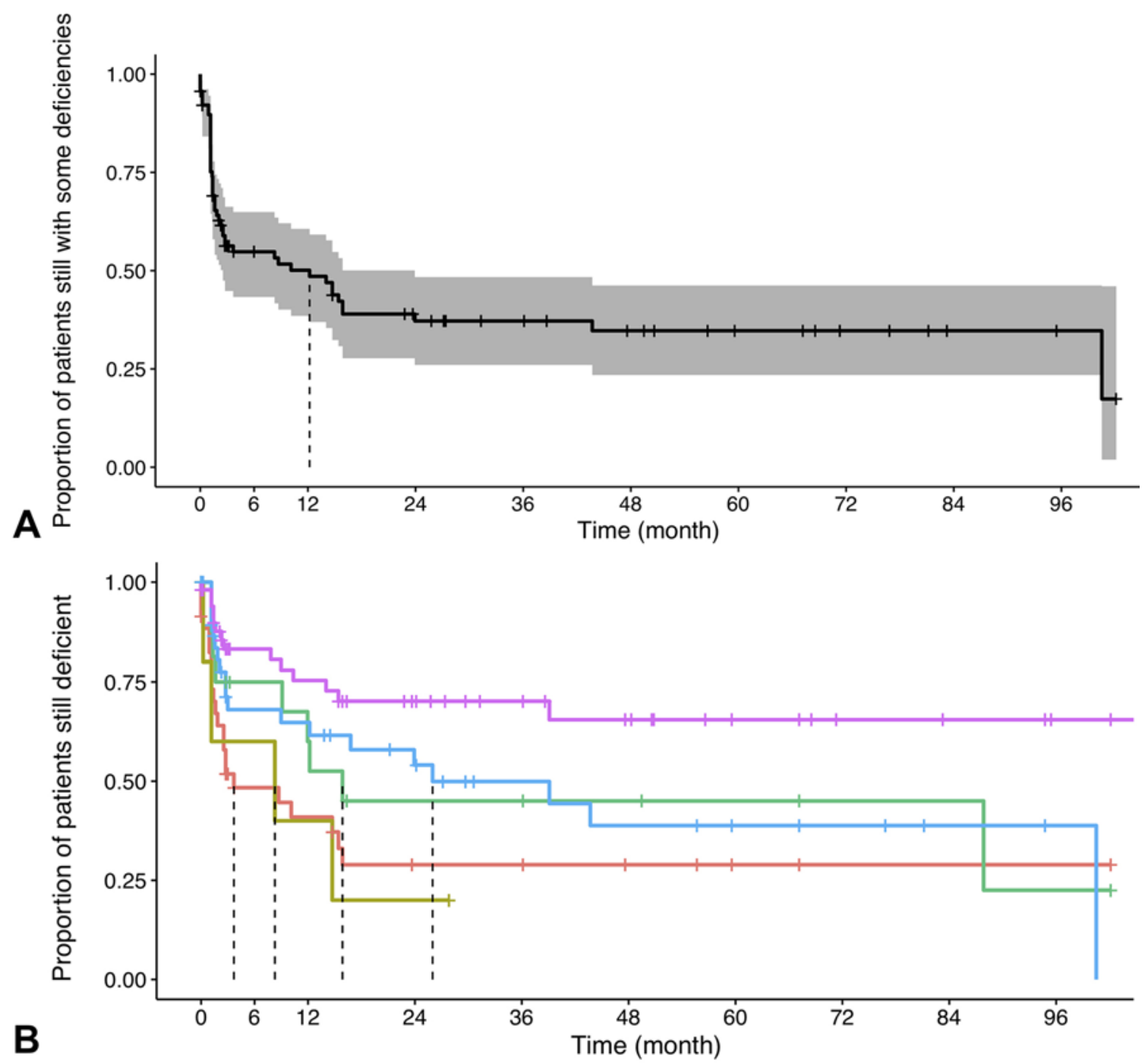

Number at risk

$\begin{array}{ccccc}35 & 6 & 4 & 1 & 1 \\ 6 & 1 & 0 & 0 & 0 \\ 17 & 5 & 3 & 2 & 1 \\ 40 & 13 & 7 & 4 & 1 \\ 52 & 21 & 12 & 5 & 2\end{array}$

Strata + Axis=Adrenocorticoid + Axis=Female gonadotrope + Axis=Growth hormone + Axis=Male gonadotrope + Axis=Thyroid

FIG. 2. Kaplan-Meier curves. A: Time to the earliest postoperative recovery of any anterior pituitary endocrine deficit. In patients with a single preoperative deficit, the time to recovery of that deficit was used. In patients with multiple preoperative deficits, the time to recovery of the earliest recovering deficit was used. Time $=0$ represents the day of surgery. Error bands represent $95 \%$ Cls. B: Time to recovery of endocrine function by affected axis. Hypothyroidism was slower to resolve relative to adrenal insufficiency $(p<0.001)$, female hypogonadism $(p<0.01)$, and male hypogonadism $(p<0.05)$. The time to recovery of the remaining axes was not significantly different from one another. Dashed lines represent median time to recovery for that axis. Figure is available in color online only.

at the endocrinologist's discretion. This definition of endocrine recovery may underestimate both the rate of and time to postoperative endocrine recovery. In older patients, in whom the expectation of recovery is lower, treating clinicians may be less inclined to attempt to wean patients off HRT, which may subsequently lead to an overestimation of the effect of age on the likelihood of endocrine recovery. In particular, this may affect the recorded rate of resolution of hypothyroidism because older patients may have baseline insufficiency of the thyroid gland, which may compound any central hypothyroidism, and therefore make it more difficult to discontinue thyroid hormone replacement therapy. ${ }^{20,22}$ Given these limitations, the rate and time to postoperative endocrine recovery reported here represent estimates in a real-life setting that are influenced both by biology (i.e., recovery of glandular function) and clinical 
TABLE 4. Incidence of new endocrine deficits postoperatively

\begin{tabular}{|c|c|c|c|c|}
\hline \multirow[b]{2}{*}{ New Endocrine Deficit } & \multicolumn{2}{|r|}{ Transient } & \multicolumn{2}{|c|}{ Persistent at LTFU } \\
\hline & No. of Pts (\%) & Median Time to Recovery, mos (IQR) & No. of Pts $(\%)$ & Median FU Time, mos (IQR) \\
\hline Diabetes insipidus & $8(5.0)$ & $0.2(0.1-0.6)$ & $4(2.5)$ & $26.8(17.9-32.3)$ \\
\hline Hypothyroidism & $0(0)$ & - & $10(9.3)$ & $38.3(19.9-70.0)$ \\
\hline Adrenal insufficiency & $12(9.6)$ & $2.5(0.6-12.2)$ & $9(7.2)$ & $49.5(25.8-68.6)$ \\
\hline Female hypogonadism & $0(0)$ & - & $0(0)$ & - \\
\hline Male hypogonadism & $1(2.3)$ & 20.9 & $2(4.5)$ & $66.0(54.2-77.9)$ \\
\hline GH deficiency & $0(0)$ & - & $4(2.8)$ & $62.3(51.0-74.0)$ \\
\hline
\end{tabular}

FU = follow-up; LTFU = long-term FU.

Denominators for percentages are number of patients without the deficit preoperatively.

management (i.e., endocrinologists' likelihood to attempt a trial off HRT).

\section{Tumor Size and Gland Volume}

Larger tumor size has long been known to increase the likelihood of hormone dysfunction in a variety of sellar pathologies. ${ }^{11-13,19}$ In this study, larger tumors were indeed significantly associated with preoperative endocrine dysfunction on bivariate analysis. On multivariable analysis, controlling for patient age and sex, this association was not maintained. This may be due to the fact that only macroadenomas were included in this study. It is possible that once the NFPA is larger than $10 \mathrm{~mm}$, other patient characteristics contribute more significantly to preoperative endocrine deficits.

The results of volumetric analysis focusing on gland volume have been variable. In their series of both functioning and nonfunctioning macroadenomas, Cho et al. ${ }^{6}$ reported in 2016 that smaller gland size (defined as its maximal thickness at the level of stalk insertion) was associated with higher odds of preoperative endocrine dysfunction, controlling for age, sex, and tumor subtype, among other factors.

TABLE 5. Volumetric analysis of the pituitary stalk and gland in 72 patients

\begin{tabular}{lc}
\hline \multicolumn{1}{c}{ Variable } & Value \\
\hline Median tumor diameter, $\mathrm{mm}(\mathrm{IQR})$ & $21.05(17.75-25.63)$ \\
\hline Median gland vol, $\mathrm{cm}^{3}(\mathrm{IQR})$ & \\
\hline Preop & $0.18(0.13-0.28)$ \\
\hline Postop & $0.33(0.23-0.48)$ \\
\hline Median \% change in gland vol (IQR) & $75.55(5.94-154.73)$ \\
\hline Preop stalk deviation, $\mathrm{n}(\%)$ & $24(33.8)$ \\
\hline Midline & $47(66.2)$ \\
\hline Deviated & $28(59.6)$ \\
\hline Postop normalization of stalk position, $\mathrm{n}(\%)$ & $19(40.4)$ \\
\hline Returned to midline & \\
\hline Still deviated & $3.30(2.50-3.80)$ \\
\hline Median stalk diameter, mm (IQR) & $3.30(3.00-3.70)$ \\
\hline Preop & \\
\hline Postop
\end{tabular}

They additionally found that the reexpansion of the gland between the immediate postoperative scan and a scan at 3 months time was associated with endocrine recovery at a median 12.3 months follow-up. In 2013, however, Cho et al. ${ }^{5}$ found no relationship between compression of the gland and endocrine status, also assessing gland size according to its thickness in a range of sellar pathologies. Focusing on NFPAs, Jahangiri et al. found that smaller preoperative gland volumes (assessed via 3D segmentation) were not associated with preoperative endocrine dysfunction, but were associated with a lower likelihood of postoperative endocrine recovery. ${ }^{13}$ Postoperative gland volume was not associated with endocrine function in that study.

In this study, neither gland volume nor postoperative gland expansion at long-term follow-up of $\geq 6$ months (median 24.4 months) was associated with the incidence of preoperative endocrine dysfunction or the likelihood of recovery. Smaller postoperative gland volume was associated with the patient's likelihood of having new postoperative anterior pituitary deficits. Similar to prior reports, we observed significant postoperative gland expansion. ${ }^{6,13}$ It is unclear whether this expansion represents restoration of normal secretory tissue or rather is stromal expansion in response to postoperative inflammation.

Others have noted the difficulty in delineating the normal gland in the presence of sellar pathology distorting the normal anatomy, even with advanced imaging. $5,6,8,13$ This may be particularly challenging when the gland is compressed to a thin layer overlaying the adenoma. These factors may be compounded by variability in imaging and segmentation techniques used across studies, and altogether contribute to the variability in reported results in NFPA volumetric studies thus far. Additionally, we observed significant interpatient variability in gland volume both preand postoperatively. This is unsurprising given the wellestablished baseline variability in pituitary size based on age and sex, even in the absence of pathology; this poses a fundamental challenge to pooled volumetric analysis in the absence of comparison to age- and sex-specific standards. ${ }^{9,26}$ At present, the only reproducible observations in the literature with regard to volumetric analysis in patients with NFPA are that larger tumor size contributes to endocrine dysfunction, and that the gland tends to expand postoperatively (not taking into account cases of apoplexy), although the clinical significance of the latter is still unclear. 


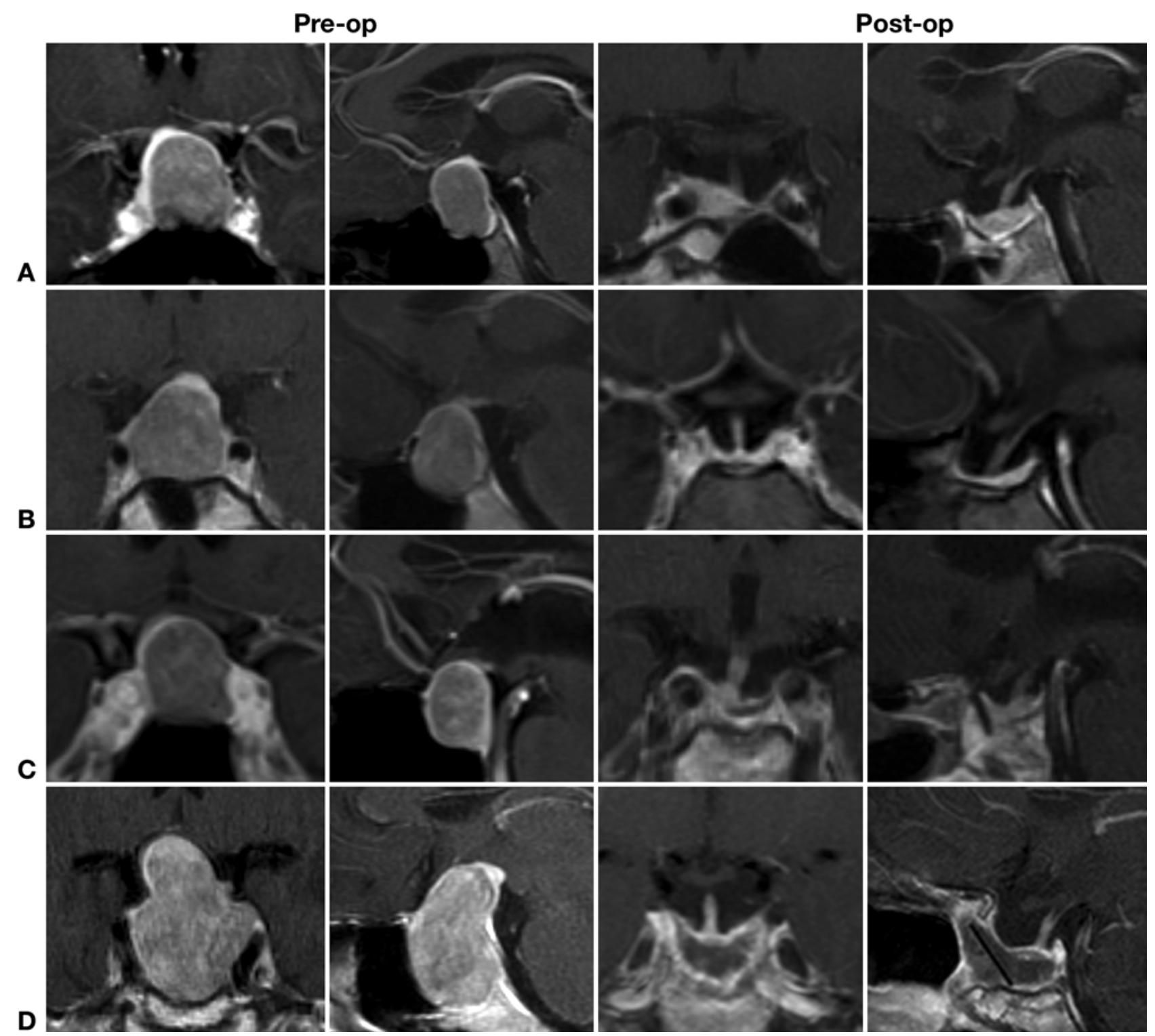

FIG. 3. Pre- and postoperative coronal and sagittal T1-weighted contrast-enhanced images of the sella. A: Images from a 29 -year-old woman with no preoperative endocrine deficits. Gland volume was above average both pre- and postoperatively, with expansion of $152 \%$. B: Images obtained in a 22 -year-old woman with no preoperative endocrine deficits. Gland volume was below average both pre- and postoperatively, with expansion of $87 \%$. C: Images from a 65 -year-old man with preoperative hypothyroidism and hypogonadism. Only the hypothyroidism recovered postoperatively. Gland volume was below average both pre- and postoperatively, with expansion of $27 \%$. D: Images obtained in a 52-year-old man with preoperative hypothyroidism, adrenal insufficiency, and hypogonadism. Only the adrenal insufficiency recovered postoperatively. Gland volume was above average preoperatively and marginally below average postoperatively, with a decrease in volume of $15 \%$.

\section{Conclusions}

In the current study we demonstrated the differential susceptibility of the different anterior pituitary hormone axes to dysfunction in the presence of NFPAs, as well as their differential likelihood to recover following endoscopic transsphenoidal resection. This is the largest known series to date to report on a homogeneous population with long-term radiological and endocrine follow-up. This paper further expands on the time course of postoperative recovery. Volumetric analysis of the gland was not found to be significantly associated with the presence of preop- erative endocrine dysfunction or its recovery. Further work may delineate whether gland expansion following decompression entirely represents normal, potentially functioning glandular tissue.

\section{References}

1. Arafah BM: Reversible hypopituitarism in patients with large nonfunctioning pituitary adenomas. J Clin Endocrinol Metab 62:1173-1179, 1986

2. Arafah BM, Kailani SH, Nek1 KE, Gold RS, Selman WR: Immediate recovery of pituitary function after transsphenoi- 
dal resection of pituitary macroadenomas. J Clin Endocrinol Metab 79:348-354, 1994

3. Briet C, Salenave S, Bonneville JF, Laws ER, Chanson P: Pituitary apoplexy. Endocr Rev 36:622-645, 2015

4. Chen L, White WL, Spetzler RF, Xu B: A prospective study of nonfunctioning pituitary adenomas: presentation, management, and clinical outcome. J Neurooncol 102:129-138, 2011

5. Cho CH, Barkhoudarian G, Hsu L, Bi WL, Zamani AA, Laws ER: Magnetic resonance imaging validation of pituitary gland compression and distortion by typical sellar pathology. J Neurosurg 119:1461-1466, 2013

6. Cho KR, Hong SD, Hur KY, Kim ST, Seol HJ, Lee JI, et al: Serial re-expansion of pituitary gland is associated with endocrinologic recovery. World Neurosurg 90:496-503, 2016

7. Dallapiazza RF, Grober Y, Starke RM, Laws ER Jr, Jane JA Jr: Long-term results of endonasal endoscopic transsphenoidal resection of nonfunctioning pituitary macroadenomas. Neurosurgery 76:42-53, 2015

8. Di Maio S, Biswas A, Vézina JL, Hardy J, Mohr G: Pre- and postoperative magnetic resonance imaging appearance of the normal residual pituitary gland following macroadenoma resection: Clinical implications. Surg Neurol Int 3:67, 2012

9. Doraiswamy PM, Potts JM, Axelson DA, Husain MM, Lurie $\mathrm{SN}, \mathrm{Na} \mathrm{C}$, et al: MR assessment of pituitary gland morphology in healthy volunteers: age- and gender-related differences. AJNR Am J Neuroradiol 13:1295-1299, 1992

10. Ebersold MJ, Quast LM, Laws ER Jr, Scheithauer B, Randall $\mathrm{RV}$ : Long-term results in transsphenoidal removal of nonfunctioning pituitary adenomas. J Neurosurg 64:713-719, 1986

11. Fatemi N, Dusick JR, Mattozo C, McArthur DL, Cohan P, Boscardin J, et al: Pituitary hormonal loss and recovery after transsphenoidal adenoma removal. Neurosurgery 63:709719, 2008

12. Iglesias $P$, Arcano K, Triviño V, García-Sancho P, Díez JJ, Villabona C, et al: Prevalence, clinical features, and natural history of incidental clinically non-functioning pituitary adenomas. Horm Metab Res 49:654-659, 2017

13. Jahangiri A, Wagner JR, Han SW, Tran MT, Miller LM, Chen R, et al: Improved versus worsened endocrine function after transsphenoidal surgery for nonfunctional pituitary adenomas: rate, time course, and radiological analysis. J Neurosurg 124:589-595, 2016

14. Jane JA Jr, Laws ER Jr: The management of non-functioning pituitary adenomas. Neurol India 51:461-465, 2003

15. Laws ER Jr, Iuliano SL, Cote DJ, Woodmansee W, Hsu L, Cho CH: A benchmark for preservation of normal pituitary function after endoscopic transsphenoidal surgery for pituitary macroadenomas. World Neurosurg 91:371-375, 2016

16. Mindermann T, Wilson CB: Age-related and gender-related occurrence of pituitary adenomas. Clin Endocrinol (Oxf) 41:359-364, 1994

17. Molitch ME: Diagnosis and treatment of pituitary adenomas: a review. JAMA 317:516-524, 2017

18. Mortini P, Barzaghi R, Losa M, Boari N, Giovanelli M: Surgical treatment of giant pituitary adenomas: strategies and results in a series of 95 consecutive patients. Neurosurgery 60:993-1004, 2007
19. Nomikos P, Ladar C, Fahlbusch R, Buchfelder M: Impact of primary surgery on pituitary function in patients with nonfunctioning pituitary adenomas - a study on 721 patients. Acta Neurochir (Wien) 146:27-35, 2004

20. Peeters RP: Subclinical hypothyroidism. N Engl J Med 376:2556-2565, 2017

21. Penn DL, Burke WT, Laws ER: Management of non-functioning pituitary adenomas: surgery. Pituitary 21:145-153, 2018

22. Sawin CT, Castelli WP, Hershman JM, McNamara P, Bacharach P: The aging thyroid. Thyroid deficiency in the Framingham Study. Arch Intern Med 145:1386-1388, 1985

23. Scangas GA, Laws ER Jr: Pituitary incidentalomas. Pituitary 17:486-491, 2014

24. Sinha S, Sharma BS: Giant pituitary adenomas-an enigma revisited. Microsurgical treatment strategies and outcome in a series of 250 patients. Br J Neurosurg 24:31-39, 2010

25. Thapar K, Kovacs K, Laws ER: The classification and molecular biology of pituitary adenomas. Adv Tech Stand Neurosurg 22:3-53, 1995

26. Tsunoda A, Okuda O, Sato K: MR height of the pituitary gland as a function of age and sex: especially physiological hypertrophy in adolescence and in climacterium. AJNR Am J Neuroradiol 18:551-554, 1997

27. Vargas G, Gonzalez B, Ramirez C, Ferreira A, Espinosa E, Mendoza V, et al: Clinical characteristics and treatment outcome of 485 patients with nonfunctioning pituitary macroadenomas. Int J Endocrinol 2015:756069, 2015

28. Zaidi HA, Cote DJ, Castlen JP, Burke WT, Liu YH, Smith TR, et al: Time course of resolution of hyperprolactinemia after transsphenoidal surgery among patients presenting with pituitary stalk compression. World Neurosurg 97:2-7, 2017

\section{Disclosures}

The authors report no conflict of interest concerning the materials or methods used in this study or the findings specified in this paper.

\section{Author Contributions}

Conception and design: Laws, Harary, Smith, Zaidi. Acquisition of data: Harary, DiRisio, Dawood, Kim, Lamba. Analysis and interpretation of data: Harary, DiRisio, Dawood, Cho, Smith, Zaidi. Drafting the article: Harary, DiRisio. Critically revising the article: Laws, Harary, DiRisio, Lamba, Smith, Zaidi. Reviewed submitted version of manuscript: Laws, Harary, DiRisio, Dawood, Kim, Smith, Zaidi. Statistical analysis: Harary, DiRisio. Study supervision: Laws, Smith, Zaidi.

\section{Correspondence}

Edward R. Laws Jr.: Brigham and Women's Hospital, Boston, MA.elaws@bwh.harvard.edu. 